\title{
Construction of a High-Quality Yeast Two-Hybrid Library and Its Application in Identification of Interacting Proteins with Brn1 in Curvularia lunata
}

\author{
Jin-Xin Gao ${ }^{1,2, \uparrow}$, Jing Jing ${ }^{1,2, \uparrow}$, Chuan-Jin Y $\mathbf{u}^{1,2}$ and Jie Chen ${ }^{1,2 *}$ \\ ${ }^{1}$ School of Agriculture and Biology, Shanghai Jiaotong University, Shanghai 200240, P. R. China \\ ${ }^{2}$ State Key Laboratory of Microbial Metabolism, Shanghai Jiaotong University, Shanghai 200240, P. R. China
}

(Received on January 1, 2015; Revised on April 1, 2015; Accepted on April 7, 2015)

Curvularia lunata is an important maize foliar fungal pathogen that distributes widely in maize growing area in China, and several key pathogenic factors have been isolated. An yeast two-hybrid (Y2H) library is a very useful platform to further unravel novel pathogenic factors in C. lunata. To construct a high-quality full length-expression cDNA library from the $C$. lunata for application to pathogenesis-related protein-protein interaction screening, total RNA was extracted. The SMART (Switching Mechanism At 5' end of the RNA Transcript) technique was used for cDNA synthesis. Double-stranded cDNA was ligated into the pGADT7Rec vector with Herring Testes Carrier DNA using homologous recombination method. The ligation mixture was transformed into competent yeast AH109 cells to construct the primary cDNA library. Eventually, a high qualitative library was successfully established according to an evaluation on quality. The transformation efficiency was about $6.39 \times 10^{5}$ transformants $/ 3 \mu \mathrm{g}$ pGADT7-Rec.The titer of the primary cDNA library was $2.5 \times 10^{8} \mathrm{cfu} / \mathrm{mL}$. The numbers for the cDNA library was $2.46 \times 10^{5}$. Randomly picked clones show that the recombination rate was $\mathbf{8 8 . 2 4 \%}$. Gel electrophoresis results indicated that the fragments ranged from 0.4 kb to $3.0 \mathrm{~kb}$. Melanin synthesis protein Brn1 (1,3,8-hydroxynaphthalene reductase) was used as a "bait" to test the sufficiency of the $\mathrm{Y} 2 \mathrm{H}$ library. As a result, a cDNA clone encoding VelB protein that was known to be involved in the regulation of diverse cellular processes, including control of secondary metabolism containing melanin and toxin production in many filamentous fungi was identified. Further study on the exact role of the $V e l B$ gene is underway.

\footnotetext{
These authors contributed equally to this work.

*Corresponding author.

Phone) +86-21-34206141, FAX) +86-21-34206141

E-mail)jiechen59@sjtu.edu.cn
}

Keywords : Brn1, Curvularia lunata, pathogenesis-related protein, VelB, Y2H library

The filamentous fungus Curvularia lunata (Wakker) Boedijn (telemorph: Cochliobolus lunatus R. R. Nelson \& F. A. Haasis) is a ubiquitous plant pathogen, which is causal agent of Curvularia leaf spot (CLS) (Macri \& Lenna, 1974). Infection of maize leaves with $C$. lunata leads to huge yield loss in severe epidemic years in northern China. For instance, it occurred over $192,000 \mathrm{hm}^{2}$ and led to 8 million $\mathrm{kg}$ yield loss in Liaoning province in 1996 (Dai et al., 1995; Dai et al., 1998). Because of nationwide extensive application of resistance varieties containing tropic and sub-tropic germplasms, the incidence of disease infection and its severity were declined massively and less damage was observed in field. However, in recent years, the disease has bounced back again and caused serious damages in some maize growing areas such as Liaoning, Henan and Anhui province etc (Gao et al., 2014b). More importantly, methyl 5-(hydroxymethyl) furan-2-carboxylate (M5HF2C) produced by $C$. lunata in infected grains pose a serious threat to human and animal health (Gao et al., 2014a). Despite the high economic impact of CLS, efficient strategies for the management of CLS have not been fully developed, which could result partially from our limited knowledge on C. lunata pathogenomics.

In the last two decades, significant progress has been made towards a better understanding of the factors involved in the pathogenesis of $C$. lunata. A multiple virulence factors have been demonstrated to be involved in pathogen infection to maize, such as cellulose (Feng et al., 2002), non-host specific toxin (methyl 5-(hydroxymethyl)furan-2-carboxylate) (Liu et al., 2009), melanin (Xu et al., 2007). It is worth mentioning that some of virulence related genes have been successfully cloned in previous work such as Brn1 being required for DHN melanin synthesis (Liu et al., 2011), Clt-1 regulating non-host specific toxin pro- 
duction (Gao et al., 2015), two mitogen-activated protein kinases (MAPK) encoding genes (Clkl and Clm1) (Gao et al., 2012; Wang and Chen, 2011). More recently, it has been reported that the Brnl gene is not only responsible for melanin synthesis but also contributes, to some extent, to toxin production. Whereas, the related pathogenicity genes in melanin and toxin production have not been extensively studied.

A proteomic approach allows the simultaneous characterization of a large number of relevant proteins expressed by a genome (Conrad et al., 2008). Using the proteomic approach, researchers have successfully identified a Clt1-regulated protein scytalone dehydratase (SCD, accession: KJ507954) associated with the production of toxin and pathogenicity in C. lunata (Gao et al., 2015). These studies are crucial in understanding the regulating network underlying pathogen virulence. However, the information presented in these studies can not directly reveal the proteins action mechanism of disease's pathogenicity. Information on pathogenic factor interrelation and their interactions on each other is required for developing strategies to prevent fungal infection. Protein interaction networks are of fundamental importance to almost all biological processes, a powerful and high-throughput protein-protein $\mathrm{Y} 2 \mathrm{H}$ screen system becomes more and more crucial to identify or mine the partners of proteins in the regulatory complexes (Miller and Stagljar, 2004).

To screen the pathogenesis-related interaction protein, an efficient and high-quality full-length cDNA library is necessary for studying the protein function and finding the protein associated with the pathopoiesia mechanism against host leaf. This study described a method for constructing a full-length cDNA library of $C$. lunata using the switching mechanism at the 5'-end of RNA transcript (SMART) technology. Moreover, melanin synthesis protein 1,3,8-hydroxynaphthalene reductase (Brn1 gene, GeneBank accession number: DQ358052) was used as a "bait" to test the efficiency of the Y2H library. This study not only demonstrated the critical techniques to construct a high-quality Y2H library but also provided informative cues to unravel interacting proteins with Brn1 involved in multiple secondary metabolic pathways in C. lunata.

\section{Materials and Methods}

Fungal strains used for the Y2H library. C. lunata CX-3 strain was selected for constructing cDNA library since it is highly virulent to maize and caused typical lesion on the maize leaves. The monoconidial culture of $C$. lunata was grown on potato dextrose agar (PDA) medium at $28^{\circ} \mathrm{C}$
(Gao et al., 2014a). The strain is stored in our laboratory and used for the study of pathogenicity mechanism of the pathogen for 10 years. The strain is maintained on PDA medium at $4^{\circ} \mathrm{C}$ or silicone beads at $-20^{\circ} \mathrm{C}$.

cDNA library primer. Universal primers were designed to amplify the inserted fragments of the cDNA library according to vector pGADT7-Rec sequence, and synthesized by Shanghai Sangon Company (Shanghai, China) as follows: MATCHMAKER 5' AD LD-Insert Screening Amplimer: 5'-CTATTCGATGATGAAGATACCCCACCAAACCC-3'; MATCHMAKER 3' AD LD-Insert Screening Amplimer: 5'-GTGAACTTGCGGGGTTTTTCAGTATCTACGATT-3'.

Total RNA isolation. To extract fungal RNA, the strains were grown in $100 \mathrm{ml}$ of PD medium in 250-ml Erlenmeyer flasks with continuous shaking at $160 \mathrm{rpm}$ for $72 \mathrm{~h}$ at $28^{\circ} \mathrm{C}$. The mycelia produced from these cultures were harvested by filtration using three layers of sterile cheese cloth, washed with sterilized double-distilled (dd) $\mathrm{H}_{2} \mathrm{O}$, and ground in liquid nitrogen. Total RNA was extracted using Trizol Reagent (Invitrogen, Carlsbad, CA) from the powdered mycelia according to the manufacturer's instructions, and then treated with DNaseI (Takara, Japan) to remove eliminate contaminated genomic DNA. The integrity of total RNA was analyzed by $1 \%$ agarose gel electrophoresis, followed by ethidium bromide $(\mathrm{EtBr})$ staining. The concentration and purity of total RNA were determined by a spectrophotometer (Eppendorf AG, Hamburg, Germany) at 260 and $280 \mathrm{~nm}$, respectively.

cDNA synthesis. The single-stranded cDNA (sscDNA) were synthesized using a SMART ${ }^{\mathrm{TM}}$ cDNA Library Construction Kit (Clontech USA, Mountain View, CA) according to the manufacturer's instructions. sscDNA were synthesized by $1 \mu \mathrm{g}$ of total RNA using SMART III oligo (5'-AAGCAGTGGTATCAACGCAGAGTGGCCATTATGGCCGGG-3'), CDS III primer (5'-ATTCTAGAGGCCGAGGCGGCCGACATG-d(T) $\left.{ }_{30} \mathrm{VN}-3^{\prime}\right)$, and Moloney Murine Leukemia Virus (MMLV) Reverse Transcriptase. Double-stranded cDNA (dscDNA) were acquired by 20 cycles of long-distance PCR using Advantage 2 Polymerase Mix (Clontech USA, Mountain View, $\mathrm{CA}$ ) with $2 \mu \mathrm{l}$ of sscDNA product. The reaction parameters were as follows: pre-thermal denaturation at $95^{\circ} \mathrm{C}$ for $30 \mathrm{~s}$; followed by 20 cycles of $95^{\circ} \mathrm{C}$ for 10 (extension/ enhance $5 \mathrm{~s}$ after each cycle), and $68^{\circ} \mathrm{C}$ for $6 \mathrm{~min}$. To identify the quality of dscDNA, $5 \mu$ of the PCR products was analyzed on $1 \%$ agarose gel electrophoresis, followed by 
EtBr staining. To get rid of low-molecular-weight dscDNA fragments, small DNA contaminants, and unincorporated nucleotides from dscDNA, the PCR products were purified by a CHROMA SPIN ${ }^{\mathrm{TM}}$ TE-400 column (Clontech USA, Mountain View, CA). The purified dscDNA were quantified by electrophoresis on $1 \%$ agarose/EtBr gel.

Construction and evaluation of the cDNA library. The purified dscDNA, together with linearized pGADT7-Rec AD cloning vector (Clontech USA, Mountain View, CA), were co-transformed into yeast competent cell AH109, where yeast repair enzymes restore the linearized plasmid to its circular form by recombining homologous sequences at the end of the dscDNA and pGADT7-Rec. The welldistributed yeast cells were then cultured on 100 dropout media (SD/-Leu) $150 \mathrm{~mm}$ plates, and incubated at $30^{\circ} \mathrm{C}$ until clones appeared (3-6 d). All clones were pooled with freezing medium (YPDA medium with $25 \%$ glycerol) and stored at $-80^{\circ} \mathrm{C}$. Transformed yeast cells $(1 \mathrm{ml})$ were diluted with YPDA medium, and $100 \mu \mathrm{l}$ was spread in dilutions of $10^{-1}, 10^{-2}, 10^{-3}, 10^{-4}$, and $10^{-5}$ dilutions on $150 \mathrm{~mm} \mathrm{SD} /-$ Leu plates, respectively. After culturing at $30^{\circ} \mathrm{C}$ for $3-6 \mathrm{~d}$, the positive transformants were harvested to form an $\mathrm{Y} 2 \mathrm{H}$ library. The transformation efficiency and library titer were calculated by counting the clones (cfu) as follows: transformation efficiency $=$ Colonies on $\mathrm{SD} /-$ Leu $\times$ dilution factor $\div$ volume $(\mathrm{ml})$ plated $\times 6 \mathrm{ml}$ per $3 \mu \mathrm{g}$ pGADT7-Rec; library titer $(\mathrm{cfu} / \mathrm{ml})=$ Colonies on $\mathrm{SD} /-$ Leu $\times$ dilution factor $\div$ volume $(\mathrm{ml})$ plated. The colonies were randomly selected to identify the size of the inserting cDNA fragment and calculate the reorganization ratio using MATCHMAKER AD LD-Insert primers based on the following PCR conditions: $94^{\circ} \mathrm{C}$ for $3 \mathrm{~min} ; 30$ cycles of $94^{\circ} \mathrm{C}$ for 30 $\mathrm{s}, 55^{\circ} \mathrm{C}$ for $30 \mathrm{~s}, 72^{\circ} \mathrm{C}$ for $1 \mathrm{~min}$; and $72^{\circ} \mathrm{C}$ for $10 \mathrm{~min}$. The PCR product $(5 \mu \mathrm{l})$ was load on a $1 \%$ agarose/EtBr gel. Recombination rate $=$ reaction number with cDNA fragments $/$ total reaction number $\times 100 \%$.

C. lunata $\mathrm{Y} 2 \mathrm{H}$ library application for the Brn1 protein interaction. In this study, Brn1 (melanin synthesis protein 1,3,8-hydroxynaphthalene (THN) reductase) was selected to detect the sufficiency of the resultant $\mathrm{Y} 2 \mathrm{H}$ library. The coding sequence of Brnl encoding 267-amino acid were amplified from the cDNA of $C$. lunata. The cDNA fragment of Brnl was inserted into the EcoRI-BamHI sites of the yeast GAL4 binding domain vector pGBKT7 (Clontech, Mountain View, CA, USA) to generate BD-bait plasmid (pGBKT7-Brn1). Subsequently, the fusion construct was transformed into the yeast strain Y187 and the positive colonies were selected on SD/-Trp medium. Furthermore, the toxicity and autotranscriptional activation of the fusion construct were tested on SD/-Trp/+Kan (20 ug/ml) liquid medium and $\mathrm{SD} /-\mathrm{Ade} /-\mathrm{Trp} /+\mathrm{X}-\alpha-\mathrm{GAL}$ plate, respectively. The $\mathrm{Y} 2 \mathrm{H}$ AD fusion library was screened using the pGBKT7-Brn1 vector in yeast-mating way. The $C$. lunata library strain (AH109) with Brn1-bait-expressing reporter strain (Y187) were co-cultured, without time-consuming and labor-intensive plasmid extraction as well as cotransformation steps required in traditional $\mathrm{Y} 2 \mathrm{H}$ screens. A clone from the bait transformant was mated with a clone from the prey transformant, and grown at $30^{\circ} \mathrm{C}$ overnight in $1 \mathrm{~mL}$ of yeast extract peptone dextrose broth. The mated clones were selected on SD medium fortified with both tryptophan and leucine to ensure successful mating. Finally, the interacting partners were screened on SD/-Leu /-Trp/-His/-Ade/+X- $\alpha-$ Gal.

Direct-Y2H assay. To test the auto-activation or interaction, the prey cDNA clones were co-transformed with either empty pGBKT7 vector or bait construct into AH109 strain. The co-transformants were first selected on $\mathrm{SD} /-$ $\mathrm{Leu} /-\mathrm{Trp}$ plates and later tested for the reporter gene expression on $\mathrm{SD} /-\mathrm{Leu} /-\mathrm{Trp} /-\mathrm{His} /-\mathrm{Ade} /+\mathrm{X}-\alpha-$ Gal plates.

\section{Results}

Total RNA analysis. The integrity of the total RNA, extracted with the Trzol reagent from $C$. lunata, was identified by $1 \%$ agarose gel electrophoresis. It showed three bands corresponding to ribosomal 28S, $18 \mathrm{~S}$ and 5S RNA, and there was no visible degradation (Fig. 1). The $\mathrm{OD}_{260} /$ $\mathrm{OD}_{280}$ ratio of total RNA was 1.94 with a concentration of $728.6 \mathrm{ng} / \mu \mathrm{L}$, which indicated that the total RNA had high purity and quality to construct the cDNA library.

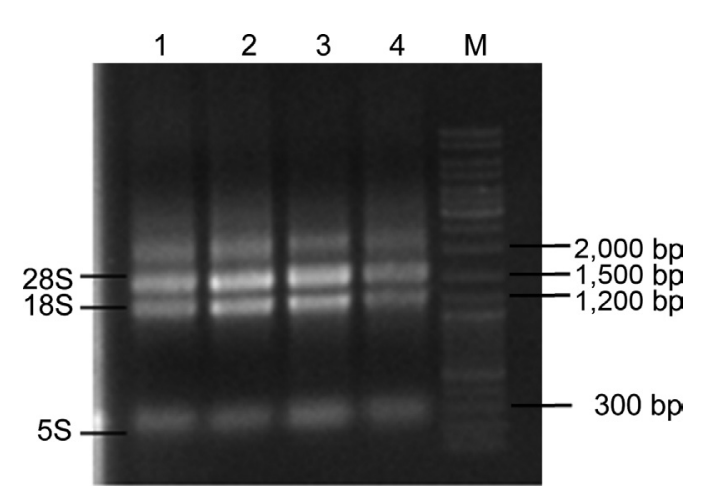

Fig. 1. Agarose gel electrophoresis of total RNA from C. lunata. Lane 1-4, total RNA (5 $\mu$ l, $728.6 \mathrm{ng} / \mu \mathrm{l})$; M, DNA Ladder Mix SM0333 (Fermentas). 


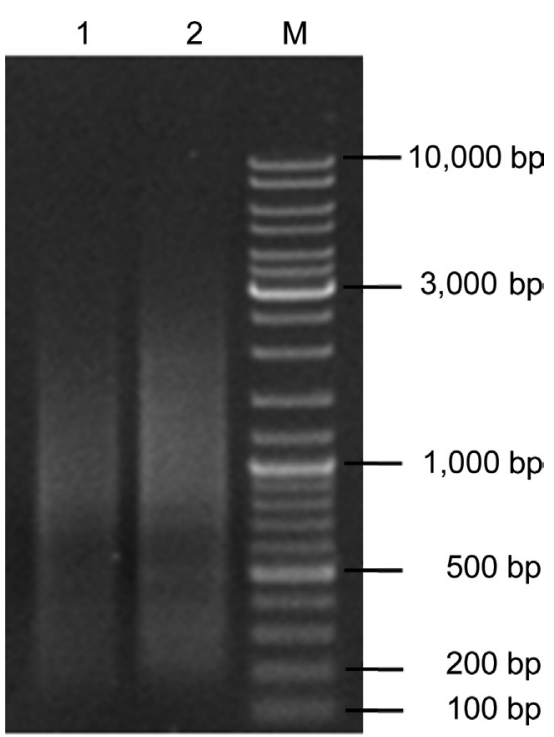

Fig. 2. Analysis of dscDNA of C. lunata on 1\% agarose gel. Lane 1, unpurified dscDNA; Lane 2, purified dscDNA; M, DNA Ladder Mix SM0333 (Fermentas).

dscDNA synthesis. dscDNA ( $5 \mu \mathrm{l})$ was analyzed by electrophoresis on $1 \%$ agarose gel. Results showed that the bands of dscDNA fragments ranged from $0.1 \mathrm{~kb}$ to $4.0 \mathrm{~kb}$ in size (Fig. 2). After purification using a Chroma Spin TE400 column, the fragments smaller than 200 bp were eliminated, which could effectively avoid the very small inserts and non-recombinant clones in the library (Fig. 2).

Construction and evaluation of the Y2H cDNA library. An Y2H cDNA library was constructed. Based on the clone number on $\mathrm{SD}$-Leu plates, the transformation efficiency was $6.39 \times 10^{5} / 3 \mu \mathrm{g}$ PGADT7-Rec and the AH109 library titer was $2.5 \times 10^{8} \mathrm{cfu} / \mathrm{ml}$, and the quantity of the cDNA

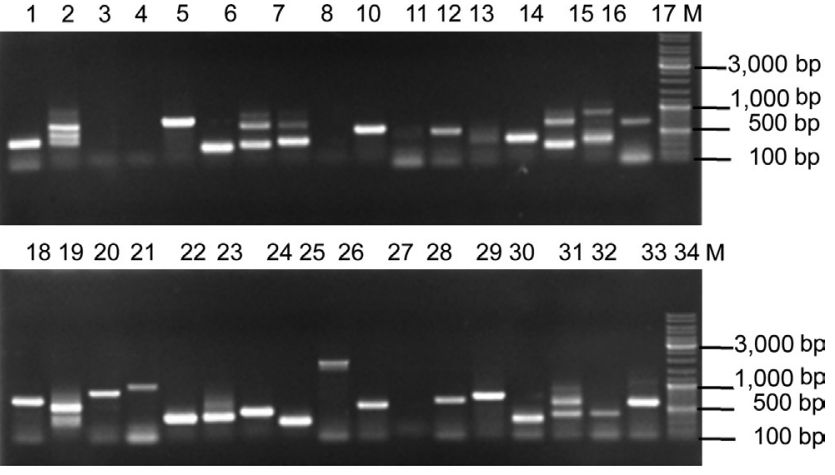

Fig. 3. The identification of the inserted dscDNA size of reading frame. 34 clones were randomly selected from SD/-Leu plates and amplified by PCR with MATCHMAKER AD LD-Insert primers. The PCR product was analyzed by $1 \%$ agarose gel electrophoresis to determine the recombination frequency and fragment size. Lanes 1-34, inserted dscDNA; M, DNA Ladder Mix SM0333 (Fermentas).

library was $2.46 \times 10^{5}$. In order to identify the length of the inserts and the recombination rate of the cDNA library, 34 positive clones were randomly selected and identified by PCR using the MATCHMAKER AD LD-Insert primers. Results showed that most of the insert ranging from 0.4 to $3.0 \mathrm{~kb}$ (Fig. 4). Most of the lanes appeared as a single band. Randomly picked clones show that the recombination rate was $88.24 \%$. The above information also indicated that the small fragment cDNA was removed from the library, and the cDNA library could be used for further research. The $\mathrm{Y} 2 \mathrm{H}$ cDNA library was harvested with freezing medium and stored at $-80^{\circ} \mathrm{C}$ for further use in Matchmaker TM gold $\mathrm{Y} 2 \mathrm{H}$ system.

Quality test of $\mathbf{Y} 2 \mathrm{H}$ screens. A total of $2.46 \times 10^{5}$ clones
A

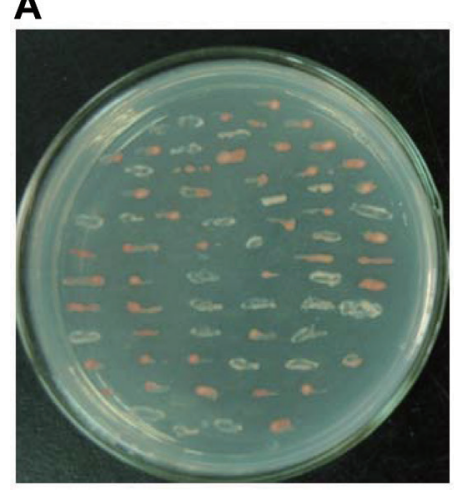

B

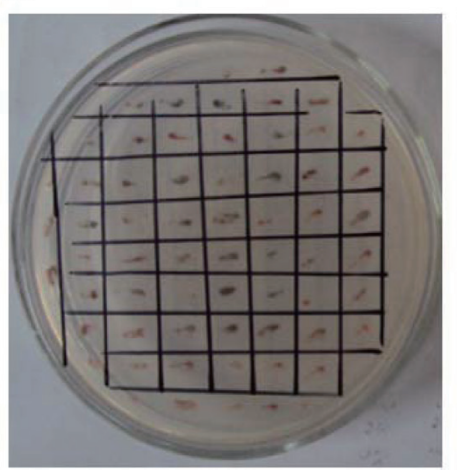

C

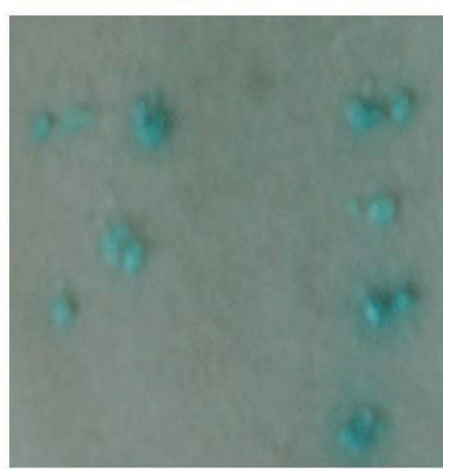

Fig. 4. Y2H screening of Brn1 protein. Yeast containing the bait (Brn1 ORF) and prey plasmid (C. lunata cDNA library) was screened for interaction on synthetic dropout media. (A) Screened for interaction on synthetic dropout SD-Trp-Leu-His-Ade media. (B) Screened for interaction on SD-Trp-Leu-His-Ade+X-Gal media. (C) Confirmation of positive interactions on SD-Trp-Leu-His-Ade+X-Gal media. 
(C. lunata cDNA Y2H library) were screened using Brn1 ORF as "bait" (Fig. 4). Among them, 3 independent clones that fulfilled the criteria of interaction with Brn1 ORF were obtained and sequenced. The sequence similarity analysis showed they were VelB, general amino acid permease Agp2 and GTPase activating protein Sec23a. To confirm interactions between Brn1 and its partners of interest, we co-transformed the corresponding DNA-binding domain (BD) and transcription-activating domain (AD) plasmids into yeast and re-identified on $\mathrm{QDO} / \mathrm{X}-\alpha-\mathrm{Gal}$. The results confirmed these proteins had genuine interactions in living cells of yeast.

\section{Discussion}

The $\mathrm{Y} 2 \mathrm{H}$ technology is by far the most powerful and widely used method to detect the protein-protein interactions in high-throughput screenings of proteomics. (Stynen et al., 2012). A high-quality expression cDNA library can provide molecular resources for analyzing the genes involved in biology and studying their protein functions and interactions facilitate the constructing of the protein network stemming from a known protein. cDNA libraries have been constructed for many plant species (Lee et al., 2012; Ma et al., 2009), animals (Han et al., 2008; Ma et al., 2011) and microorganisms (Wang and Chen, 2011; Zhao et al., 2009) and genes related to biological processes have been determined. Also, genes related to the biology of fungal pathogens, such as pathogen invasion, development, survival, pathogenicity and virulence can be discovered with a cDNA library. Few reports have been published concerning the construction of a cDNA library from C. lunata. To study the pathogenesis-related protein interaction, a fulllength cDNA library from $C$. lunata mycelia and applied it to $\mathrm{Y} 2 \mathrm{H}$ technology.

Conventional cDNA library construction methods suffer from several major shortcomings (Gubler and Hoffman, 1983). For example, the majority of cDNA clones lost whole or small fragments, especially mRNAs longer than $2 \mathrm{~kb}$. Directional cloning suffers from methylation and is often incomplete in protecting the internal restriction sites and inefficient for cloning (McClelland et al., 1994). Given the low efficiency of enzymatic enrichment steps, conventional cDNA library construction methods are also unsuitable in cases with limited starting material. As a novel and useful method for constructing a full-length cDNA library, SMART technique increases the lengths of cDNA clone and high-quality cDNA library by simultaneously employing two intrinsic properties of MMLV reverse transcriptase (template switching and reverse transcription) (Kulpa et al., 1997; Zhu et al., 2001). This method also anchors both ends of sscDNA by adding a distinct SmaI site to each end during reverse transcription. This novel integration of template switching with SmaI-based anchoring and directional cloning ensures the construction of cDNA libraries with high yields of representatives. To perform a comprehensive screen for positive interactors, it is critical that the library have as high a complexity as possible to maximize the chances of finding relevant prey proteins. Our cDNA library contained $>2.5 \times 10^{8} \mathrm{cfu} / \mathrm{mL}$ with an average insert size of $0.4-3.0 \mathrm{~kb}$, thereby ensuring a complete representation of rare sequences.

Fungal melanins are black or dark brown pigments formed by the oxidative polymerization of phenolic compounds (Bell and Wheeler, 1986). They are not considered essential for growth and development of fungal cells, but they enhance the survival and competitive abilities of fungi in certain environments (Butler \& Day, 1998). Melanin appears to contribute to virulence by reinforcing the mechanical strength of the infection into host plant epidermis in many plant diseases (Kawamura et al., 1999; Brakhage et al., 1999; Liu et al., 2011). In the maize pathogen $C$. lunata, the melanized layer of its appressoria and infection pegs, is essential for maintenance of the turgor pressure that enables effective leaf penetration. Melanin in conidia in nature primarily via a polyketide synthetase pathway from 1,3,8-trihydroxynaphthalene (THN) reductase gene $B r n 1$, associated with the melanin pathway of C. lunata, was identified and characterized in our previous study (Liu et al., 2011). The Brnl gene may also be associated with toxin production and conidiation. However, there is no detailed knowledge to reveal the genetically regulation mechanism of those virulence factors in the C. lunata so far. For this reason, we searched for more pathogenic factors, from which we could obtain more regulators that mediate melanin and toxin production, to obtain a better understanding of the regulation network that controls secondary metabolite production and conidiation.

In this study, Brn1 ORF was selected as "bait" to screen the C. lunata cDNA library. Finally, 3 proteins with positive interactions with Brn1 ORF were confirmed by sequence alignment and database retrieval. They are VelB, general amino acid permease AGP2, and GTPase activating protein Sec23a. Among these interacted proteins, VelB is our most interested protein. It carries two velvet domains located in the $\mathrm{N}$ - and C-terminal half, respectively. Velvet domain which represents a protein-protein interaction domain is found in filamentous ascomycetes and basidiomycetes (Bayram \& Braus, 2008). VelB is involved in the regulation of diverse cellular processes by interacting 
with different proteins. VelB had been described as a lightdependent regulator of fungal development and secondary metabolism of $A$. nidulans, which interacts with VeA. VelB can also form homodimers and is part of another heterodimer, VosA-VelB. VosA-VelB represses asexual development and is required for the viability of spores by activating trehalose biogenesis. In a submerged culture during vegetative growth, VosA-VelB can negatively regulate asexual conidiation and support trehalose biosynthesis (Bayram et al., 2012).

Brn1 has long been known to be closely related to the biosynthesis of melanin. However, our previous studies showed that Brnl-silenced transformants have significantly reduced toxin production from $C$. lunata. Our experiment of transcriptome analysis with consecutively induced $C$. lunata strains on resistant varieties also demonstrated that the strains with improved virulence could simultaneously up-regulate some gene expression like melanin synthesis genes, e.g. Brnl and $S C D$ genes, toxin production related genes, e.g. aflatoxin biosynthesis protein ord2 gene and HC-toxin synthetase gene (data not shown). Thus it is clear that Brn1 gene in C. lunata is closely associated with toxin biosynthesis (Liu et al., 2011). VelB is known with certain relation to fungal toxin production like sterigmatocystin (ST) in Aspergillus parasiticus, cyclopiazonic acid, aflatoxin in Aspergillus flavus, ST and $\beta$-l-catam in A. nidulans, and deoxynivalenol (DON) mycotoxin in Fusarium graminearum (Jiang et al., 2012). Therefore a question should be answered whether there is a co-regulation between Brn1 and VelB. If the co-expression of Brnl and VelB genes genuinely exists, it would be rational to consider melanin and toxin as interrelated components of a virulence entirety. The new finding of VelB associated with Brn1 would be helpful for us to globally understand the pathogen virulence mechanism.

Currently, the pathogenesis of C. lunata is not very clear. In particular, the molecular interactions of pathogenesisrelated proteins are not yet fully understood. In the current study, the Y2H system was first successful constructed and adopted to screen pathogenesis-related proteins that interact with Brn1. The exact role of $C$. lunata VelB in pathogenicity still needs to be determined by gene function analysis. Therefore, this study could serve as the starting point for a deeper analysis on the pathogenicity in C. lunata. The elucidation of this protein interaction is of much importance both in understanding the regulatory network of melanin and toxin synthesis, and as potential targets for further research on the efficient strategies for the management of CLS.

\section{Acknowledgments}

This work was supported by grants from China Agriculture Research System (CARS-02), and the National Natural Science Foundation of China (31171798, 31471734).

\section{References}

Bayram, O. and Braus, G. H. 2012. Coordination of secondary metabolism and development in fungi: the velvet family of regulatory proteins. FEMS Microbiol. Rev. 36:1-21.

Bayram, O., Krappmann, S., Ni M., Bok, J. W., Helmstaedt, K., Valerius O., Braus-Stromeyer, S., Kwon, N. J., Keller, N. P., Yu, J. H. and Braus, G. H. 2008. VelB/VeA/LaeA complex coordinates light signal with fungal development and secondary metabolism. Science 320:1504-1506.

Bell, A. A. and Wheeler, M. H. 1986. Biosynthesis and function of fungal melanins. Annu. Rev. Phytopathol. 24:411-451.

Brakhage, A. A., Langfelder, K., Wanner, G., Schmidt, A. and Jahn, B. 1999. Pigment biosynthesis and virulence. Contrib. Microbiol. 2:205-215.

Butler, M. J. and Day, A.W. 1998. Fungal melanins: a review. Can. J. Microbiol. 44:1115-1136.

Conrad, D. H., Goyette, J. and Thomas, P. S. 2008. Proteomics as a method for early detection of cancer: a review of proteomics, exhaled breath condensate, and lung cancer screening. J. Gen. Intern. Med. 23:78-84.

Dai, F. C., Gao, W. D., Wu, R. J. and Jin, X. H. 1995. A noticeable corn disease: Curvularia leaf spot. ACTA Phytopathol. Sin. 25:330.

Dai, F. C., Wang, X. M., Zhu, Z. D., Gao, W. D. and Huo, N. X. 1998. Curvularia leaf spot of maize: pathogens and varietal resistance. ACTA Phytopathol. Sin. 2:123-129.

Feng, J., Gao, Z., Xue, C., Zhuang, J., Chen, J. and Bai, S. 2002. The pathogenesis of the cell-degrading enzymes produced by Curvularia lunata. Rain Fed Crops 22:164-167.

Gao, J. X., Jing, J., Liu, T. and Chen, J. 2015. Identification of Clt-1-regulated proteins associated with the production of non-host-specific toxin and pathogenicity in Cochliobolus lunatus. J. Phytopathol. 163:33-41.

Gao, J. X., Liu, T. and Chen, J. 2014a. Insertional mutagenesis and cloning of the gene required for the biosynthesis of the non-host specific toxin in Cochliobolus lunatus that causes maize leaf spot. Phytopathology 104:332-339.

Gao, S. G., Li, Y. Q., Gao, J. X., Suo, Y. J., Fu, K. H., Li, Y. Y. and Chen, J. 2014b. Genome sequence and virulence variation-related transcriptome profiles of Curvularia lunata, an important maize pathogenic fungus. BMC Genomics 15:627.

Gao, S. G., Zhou, F. H., Liu, T., Li, Y. Y. and Chen, J. 2012. A MAP kinase gene, $C l k 1$, is required for conidiation and pathogenicity in the phytopathogenic fungus Curvularia lunata. J. Basic. Microbiol. 53:214-223. 
Gubler, U. and Hoffman, B. J. 1983. A simple and very efficient method for generating cDNA libraries. Gene 25:263-269.

Han, X. F., Luo, J., Wu, N., Matand, K., Yang, B. J., Wu, H. J., Zhang, L. J. and Wang, H. B. 2008. Construction and characterization of a goat mammary gland cDNA library. Mol. Biotechnol. 38:187-193.

Jiang, J. H., Yun, Y. Z., Liu, Y. and Ma, Z. H. 2012. FgVELB is associated with vegetative differentiation, secondary metabolism and virulence in Fusarium graminearum. Fungal Genet. Biol. 49:653-662.

Kawamura, C., Tsujimoto, T. and Tsuge, T. 1999. Targeted distribution of a melanin biosynthesis gene affects conidial development and UV Tolerance in the Japanese Pear pathotype of Aternaria alternata. Mol. Plant-Microbe Interact. 12:59-63.

Kulpa, D., Topping, R. and Telesnitsky, A. 1997. Determination of the site of first strand transfer during Moloney murine leukemia virus reverse transcription and identification of strand transfer-associated reverse transcriptase errors. $E M B O J .16$ : 856-865.

Lee, L. Y., Wu, F. H., Hsu, C. T., Shen, S. C., Yeh, H. Y., Liao, D. C., Fang, M. J., Liu, N. T., Yen, Y. C. and Dokládal, L. 2012. Screening a cDNA library for protein-protein interactions directly in planta. Plant Cell 24:1746-1759.

Liu, T., Liu, L., Jiang, X., Huang, X. and Chen, J. 2009. A new furanoid toxin produced by Curvularia lunata, the causal agent of maize Curvularia leaf spot. Can. J. Plant Pathol. 31: 22-27.

Liu, T., Xu, S., Liu, L., Zhou, F., Hou, J. and Chen, J. 2011. Cloning and characteristics of Brnlgene in Curvularia lunata causing leaf spot in maize. Eur. J. Plant Pathol. 131:211-219.

Ma, J., Huang, X., Wang, X., Chen, X., Qu, Z., Huang, L. and Kang, Z. 2009. Identification of expressed genes during compatible interaction between stripe rust (Puccinia striiformis) and wheat using a cDNA library. BMC genomics 10:586.

Ma, Y., Ruan, Q., Ji, Y., Wang, N., Li, M., Qi, Y., He, R., Sun, Z. and Ren, G. 2011. Novel transcripts of human cytomegalo- virus clinical strain found by cDNA library screening. Genet. Mol. Res. 10:566-575.

Macri, F. and Lenna, P. 1974. Leaf corn blight incited by Curvularia lunata (Wakk.) Boed. J. Plant Pathol. 10:27-35.

McClelland, M., Nelson, M. and Raschke, E. 1994. Effect of sitespecific modification on restriction endonucleases and DNA modification methyltransferases. Nucleic Acids Res. 22:36403659 .

Miller, J. and Stagljar, I. 2004. Using the yeast two-hybrid system to identify interacting proteins. Methods Mol. Biol. 261:247262.

Stynen, B., Tournu, H., Tavernier, J. and Van Dijck, P. 2012. Diversity in genetic in vivo methods for protein-protein interaction studies: from the yeast two-hybrid system to the mammalian split-luciferase system. Microbiol Mol. Biol. Rev. 76: 331-382.

Wang, J. Y. and Chen, J. 2011. Cloning and functional analysis of Clm1 in Curvularia lunata. ACTA Phytopathol Sin. 41:464472.

Wang, Y. Y., Zhang, T., Zhou, Q. M. and Wei, J. C. 2011. Construction and characterization of a full-length cDNA library from mycobiont of Endocarpon pusillum (lichen-forming Ascomycota). World J. Microb. Biot. 27:2873-2884.

Xu, S. F., Chen, J., Liu, L. X., Wang, X. F., Huang, X. L. and Zhai, Y. H. 2007. Proteomics associated with virulence differentiation of Curvularia lunata in maize in China. J. Integr. Plant Biol. 49:487-496.

Zhao, W. J., Zhang, H., Bo, X., Li, Y. and Fu, X. 2009. Generation and analysis of expressed sequence tags from a cDNA library of moniezia expansa. Mol. Biochem. Parasitol. 164: 80-85.

Zhu, Y. Y., Machleder, E. M., Chenchik, A., Li, R. and Siebert, P. D. 2001. Reverse transcriptase template switching: a SMART approach for full-length cDNA library construction. Biotechniques 30:892-897. 